\title{
La recherche à l'université de Bretagne-Sud (Lorient) de 2000 à 2007
}

\section{(2) OpenEdition \\ 1 Journals}

Édition électronique

URL : http://journals.openedition.org/abpo/249

DOI : $10.4000 / a b p o .249$

ISBN : 978-2-7535-1512-3

ISSN : 2108-6443

\section{Éditeur}

Presses universitaires de Rennes

\section{Édition imprimée}

Date de publication : 31 décembre 2008

Pagination : $315-320$

ISBN : 978-2-7535-0808-8

ISSN : 0399-0826

\section{Référence électronique}

"La recherche à l'université de Bretagne-Sud (Lorient) de 2000 à 2007 », Annales de Bretagne et des Pays de l'Ouest [En ligne], 115-4 | 2008, mis en ligne le 31 décembre 2008, consulté le 24 avril 2019. URL : http://journals.openedition.org/abpo/249; DOI : 10.4000/abpo.249

Ce document a été généré automatiquement le 24 avril 2019.

(c) Presses universitaires de Rennes 


\section{La recherche à l'université de Bretagne- Sud (Lorient) de 2000 à 2007}

1 Liste des thèses et mémoires soutenus et déposés à l'université de Bretagne-Sud.

\section{BIBLIOGRAPHIE}

\section{Maîtrises}

\section{0}

AMGHAR, Julien, Les infrastructures portuaires dans la rade de Lorient et la rivière d'Etel, dir. G. LE B OUËDEC.

AUDRAN, Karine, Les négociants lorientais sous la Révolution et l'Empire, dir. G. LE BOUËDEC.

BRAHMI, Néjat, Recherches sur le culte privé de Volubilis à l'époque romaine à travers l'étude des autels domestiques, dir. C. VENDRIES.

COLLOBERT, GWénola, La culture populaire littorale : naufrages et pillages dans l'amirauté de Quimper, 1747-1770, dir. G. LE BOUËDEC.

COSSE, Faëlan, Origines et constitution d'une Maison noble : le lignage de Rohan (xie-xiiie siècle), dir. J. QUAGHEBEUR.

CHATELIN, Laurent, Les entrepreneurs lorientais de 1800 à 1860, dir. G. LE BOUËDEC.

EVANNO, sabrine, Les sociétés des gens de mer : la femme face aux absences de l'homme dans la paroisse de Riantec et au Port-Louis, 1770-1790, dir. G. LE BOUËDEC. 
FERET, Lenaïg Les importations de céramiques à parois fines, plombifêres et métallescentes gallo-romaines à Vannes (Morbihan), dir. D. FRÈrE.

HARTEREAU, Erwan, Le port de Concarneau : mobilité des hommes et renouvellement de la flotte, dir. G. LE BOUËDEC.

KAN, Anne-Gaëlle,Le jardin botanique de Brest (1694-1820), dir. S. LLINARES.

KERNEUR, christophe, La presse catholique de Rennes de 1879 à 1906, dir. F. CHAPPÉ.

LAMOUROUX, Hélène, Les Orientaux de Volubilis à l'époque romaine, dir. C. VENDRIES.

LE BADEZET, Nicolas, Mottes et enceintes castrales dans la seigneurie du Kemenet-Heboe, dir. J. QUAGHEBEUR.

LE GLAND, Valérie, Les métiers du cuir et de la chaussure à Vannes au XVIIe et XVIIIe siècle : tanneurs et cordonniers, dir. S. LLINARES.

LE MAUX, Pierre,L'influence de l'« Etrusca disciplina » sur la religion romaine : VIe av. J.-C./Ier siècle apr. J.-C, dir. D. FRÈRE.

LE MESTRE, Dominique,La mort à l'époque mérovingienne à travers l'Histoire des Francs de Grégoire de Tours, dir. J. QUAGHEBEUR.

L'HARIDON BREAT, TYPhaine, Le mariage Tamoul à travers les archives du Tribunal de police de Pondichéry de 1788 à 1805, dir. G. LE BOUËDEC.

NICOLAS, Karine, La violence des populations migrantes et flottantes à Lorient de 1770 à 1778, dir. G. LE B OUËDEC.

PICAUD, Marie-Aude,Les imprimeurs et les libraires à Vannes et Lorient au xviiie siècle, dir. S. LLINARES.

PUJADE, Morgane, L'anticomanie dans le Morbihan (1840-1920), dir. D. FRÈRE.

QUILLERE, Michelle, Les ports de France (1829 à 1860) : Équipements, trafics et industrialisation, dir. 0. PETRE-GRENOUILLEAU.

ROLLANDO, Gaëlle, Le système sardinier de la rivière d'Etel, dir. G. LE BOUËDEC.

SPELLA, Nadège, Renaissance et crise du négoce maritime à Rostock, 1800-1820, dir. G. LE BOUËDEC.

VARY, Morgane, Le vol dans la sénéchaussée d'Hennebont au xviiie siècle, dir. G. LE BOUËDEC.

\section{1}

CARET, Gwénaëlle, La représentation des monstres en Etrurie orientalisante et archaïque, dir. D. FRÈRE. COINDET, sylvain, Les paroisses littorales face aux naufrages dans l'amirauté de Cornouaille, 1721/1740-1770/1790, dir. G. LE BOUËDEC.

COTTEN, Sandrine, La navigation et la représentation de la traversée entre la France et la Nouvelle-France d'après les récits de voyage de 1660 à 1760, dir. G. LE BOUËDEC.

DE LIGNERES, Bertrand, Les membres d'équipage et leur mobilité sur les navires de pêche et de cabotage ayant armé à Lorient entre 1785 et 1787, dir. G. LE BOUËDEC.

DREANNO, solenne, La violence dans la sénéchaussée d'Hennebont, 1770-1790, dir. G. LE BOUËDEC.

HAROCHE, Cécile, Joseph Delaville-Leroulx, négociant à Gand, 1777-1785. Le commerce intra-européen au xviiie siècle, dir. G. LE BOUËDEC. 
HoDICQ, Marie,L'abbaye Saint-Sauveur de Redon aux xie et xiie siècles : les raisons d'un cartulaire, dir. J. Q UAGHEBEUR.

KERLOC'H, Marianne, Les élites municipales de Lorient, 1738-1789, dir. G. LE BOUËDEC.

LE COROLLER, Gaël,La présence bretonne à Chartres (xie-xiie siècles), dir. J. QUAGHEBEUR.

LE GUENIC, Michel,L'image des régions dans le 13 heures de Jean-Pierre Pernaut : un nationaltraditionalisme?, dir. O. PETRE-GRENOUILLEAU.

LE GUEVEL, Emmanuelle, Le singe en Égypte : rôles fonctions et représentations de 2100 av. J.-C. au début du Ive siècle apr. J.-C., dir. C. VENDRIES.

MARZIOU, Anne-Flore,Étude de l'occupation du sol autour des vallées de l'Aff et de l'Oyon (au sud de Brocéliande) entre la fin de l'Age du Fer et l'An Mil, dir. C. VENDRIES.

NABAT, Grégory, Ria d'Etel : évolution du peuplement de l'Age du Fer à la fin de l'époque gallo-romaine (vie siècleav. J.-C.-ive siècle apr. J.-C.) ; tome : 10 : recueil bibliographique : commune de Sainte-Hélène (Morbihan), dir. D. FRÈRE.

PEREZ, Marion,La céramique des fossés de la phase 3 du site de l'Age du Fer de Kerven-Teignouse à Inguignel (Morbihan), dir. D. FRÈRE.

TIMPAGNON, stéphanie, Prosper Mérimée et la conservation des monuments gallo-romains (1834-1853), dir. C. VENDRIES.

2002

ARTUIS, Emmanuelle, Le sucré dans le pays vannetais au xviiie siècle, dir. G. LE BOUËDEC. UFFRET, Anne, Les minutes d'un notaire alréen : Maître Jacques Henry, sieur de Kernivilit (1720-1729), dir. G. LE BOUËDEC.

BARDOUILLE, Jérôme,Le génie militaire romain du Haut Empire (ier et iie siècles) : corps de métiers, réalisations, techniques, dir. C. VENDRIES.

BARTHELEMY, Magalie, Ignace Advisse-Desruisseaux : itinéraire d'un négociant vannetais au xviiie siècle, dir. G. LE BOUËDEC.

BRIENT, Xavier, Les maîtres au cabotage de Sarzeau et Saint-Gildas, 1820-1840, dir. G. LE BOUËDEC.

CADORET, vincent,L'Ouest-Eclair face au Front Populaire : mars 1936-juin 1937, dir. F. CHAPPÉ.

DANET, Marianne, Violences et comportements la nuit à Vannes, 1750-1780, dir. G. LE BOUËDEC.

FASCIANI, David, Les transformations de l'Arsenal de Lorient de 1870 à 1914, dans la perspective de la construction des grands cuirassés, dir. G. LE BOUËDEC.

HELLEC, christophe,La mémoire des voyageurs en Morbihan : représentations du littoral morbihannais au travers des relations de voyageurs extérieurs à la Bretagne de Alfred Jouvin de Rochefort à Alexandre Nicolaï, dir. G. LE BOUËDEC.

HENRIO, Marine, Les frontières de l'Acadie (1713-1763), dir. P. HRODEJ.

LE GOFF, Gildas, Une étape de l'histoire des techniques : l'étude technologique du locus 1 du site mésolithique ancien des Vingt-deux-Boissellées, Saint-Père-en-Retz (Loire-Atlantique), dir. D. FRÈRE. LHORO, Hélène,Le nationalisme breton et les Bretons : 1931-1939, dir. F. CHAPPÉ. 
MÉNARD, Marie, Transcription de la copie des correspondances entre le directeur de Lorient, Charles Bréart de Boisanger, et les directeurs généraux de la Compagnie Royales des Indes Orientales (mi-janvier au 9 novembre 1703), dir. G. LE BOUËDEC.

NERZIC, Jean-Yves, Armement en course du baron de Pointis, 1696-97, dir. P. HRODEJ.

PERRIN, Michel, Les relations de Lorient avec les pays du littoral atlantique au xviiie siècle, dir. G. LE B OUËDEC.

ROUELLE, Martine, Société et économie lorientaises sous la Révolution à travers les archives notariales, dir. G. LE BOUËDEC.

TEMPLIER, Jeanne, Collection de céramiques gallo-romaines et mérovingiennes : au musée James Miln et Zacharie Le Rouzic de Carnac, dir. M. CASSET.

TOUCHE, Erwan, Les marins face au danger : impact sur la religiosité des gens de mer du diocèse de Vannes (milieu du xviie-milieu du xixe siècle), dir. G. LE BOUËDEC.

VillemauX, Ariane,Les urnes étrusques des musées de Bretagne et des Pays de Loire : archéologie expérimentale et recherches sur les ateliers d'époque hellénistique, dir. D. FRÈRE.

\section{3}

CAGNEC, Hervé,Recherches sur la villa gallo-romaine des Bossenno à Carnac, dir. D. FRÈRE.

DANET, Mickaël,Commerce, traite et politique à Zanzibar : 1844-1905, dir. O. PETRE-GRENOUILLEAU.

DANET, Sophie, Les corsaires ou privateers du Nord-Est de l'Angleterre pendant la guerre d'Indépendance américaine (1777-1783), dir. G. LE BOUËDEC.

GUIHUR, Evelyne, Monsieur Louis Champion de Cicé,évêque in partibus infidelium de Sabule, vicaire apostolique du Siam (1701-1727), dir. G. LE BOUËDEC.

SICART, olivier, Les marins étrangers sur les navires de la Compagnie des Indes au xviiie siècle (1730-1770), dir. G. LE BOUËDEC.

FAUCHILLE, GXénaël, Des processus de patrimonialisation dans les petits ports de pêche artisanale sur la côte nord du Portugal, dir. G. LE BOUËDEC.

\section{Master 1}

\section{4}

DANIEL, Cédric,Lorient et l'Angleterre entre 1690 et 1789, dir. G. LE BOUËDEC.

GUILLOUËT, christophe,Le regard d'Arthur de La Borderie sur la Bretagne médiévale, dir. J. QUAGHEBEUR.

HALLAIS, Marc, Genèse du yachting en Bretagne atlantique : 1850-1920, dir. G. LE BOUËDEC.

LE HUNSEC, Mathieu,Reconstitution de carrières dans la marine : quartier maritime de Port-Louis, 1760-1787, dir. G. LE BOUËDEC.

LE MAUFF, Anne-Cécile, L'entretien de la route Nantes Audierne de 1753 à 1830, dir. G. LE BoUËDEC.

LE RUYET, Louis,Les ouvriers de la matricule à L'Orient de 1750 à la veille de la Révolution, dir. G. LE B OUËDEC. 
MARGOLINE-PLOT, Eugénie, La diffusion de la culture asiatique vue au travers des hardes des marins de la seconde Compagnie des Indes, dir. G. LE BOUËDEC.

MORICE, Marie-Laure, L'échec du port franc de Lorient : 1784-1790, dir. G. LE BOUËDEC.

RICHARD, Nicolas, Lorient et la Marine de 1740 à 1763 : perception, rôles, relations à travers la correspondance du ministre de la Marine avec Port-Louis, dir. G. LE BOUËDEC.

RIO, Pierre, Étude de l'occupation humaine entre l'estuaire du Blavet et la ria d'Etel du Néolithique au xiiie siècle, dir. M. CASSET.

\section{5}

BAUDET, Yannick,Les abbayes cisterciennes dans le Vannetais du XIIe au XIVe siècle : sanctuaires spirituels et puissances temporelles, dir. J. QUAGHEBEUR.

BoulIC, Ronan, Les sociétés littorales du pays de l'Aven et du Belon au XVIIIe siècle, dir. G. LE BOUËDEC.

CARTROU, André, Les élites consulaires de Vannes au XVIIIe siècle (1710-1792), dir. G. LE BOUËDEC.

FRONMAIER, Gudrun-Luise,Des «émigrés " français à Hambourg entre la Révolution et l'Empire, dir. G. LE BOUËDEC.

IACONA, Stéphane,Impact de la guerre navale sur la carrière des marins de Bretagne sud : 1793-1815, dir.

G. LE BOUËDEC.

LE BEC, Pricillia, Les impacts du canal de Nantes à Brest sur les sociétés riveraines de Malestroit et Josselin, dans la première moitié du XIXe siècle, dir. G. LE BOUËDEC.

LE BORGN, Isabelle,La vie politique vannetaise dans la tourmente de l'Histoire : 1935-1953, dir. F. CHAPPÉ.

LE BRAZIDEC, Anthony, Une ville, une rivière : le Blavet à Pontivy avant la décision de canalisation en 1802, dir. G. LE BOUËDEC.

LE QUÉRÉ, Rozenn, Société rurale et événement maritime à Plozévet : monographie d'une famille de domaniers (1700-1789), dir. G. LE BOUËDEC.

MARC-MARTIN, Alexandre, Études sur les lettres de Loup de Ferrières (805-840-862), dir. J. QUAGHEBEUR.

NOFFICIAL, Sébastien,Le port et l'arsenal de Lorient en 1793, dir. G. LE BOUËDEC.

REVAULT, stéphanie, Les Artemis d'Asie Mineure occidentale et méridionale, dir. V. MEHL.

ROMAN, Marine,La politique coloniale de la France sous le Consulat de 1799 à 1804 vue à travers «Le

Moniteur Universel », dir. O. PETRE-GRENOUILLEAU.

\section{6}

BARON, Pierre, L'Unanimisme lorientais en question (janvier 17906Juin 1791), dir. G. LE BOUËDEC

HILLION, Julien, La colonie pénitentiaire de Belle-Ile en Mer (1880-1900) : surveillance, formation professionnelle et rapports à la société locale, dir. G. LE BOUËDEC.

LANGLOIS, Ludivine, Les flottes de commerce à la pointe de Bretagne au XVIIIe siècle (d'après les dossiers de l'Amirauté de Cornouaille sur les naufrages), dir. G. LE BOUËDEC.

LE GuellanfF, Claudia, Les passagers de la Compagnie des Indes, de 1719 à 1747, d'après la sous-série 1P du Service Historique de la Marine de Lorient dir. G. LE BOUËDEC. 
2007

BAUDoIn, Marion,Les dynasties caboteuses de la Presqu'île de Rhuys au XVIIIe siècle, dir. G. LE BoUËDEC. LE PoGAM, Olivier, Les relations entre la France et la Louisiane à travers le monopole de la compagnie des Indes entre 1717 et 1732 : étude des échanges civiles : les passagers pour la Louisiane, dir. G. LE BOUËDEC. PASQUIERS-YVON, Delphine,Les pêcheurs groisillons, 1829-1835, dir. G. LE BOUËDEC.

PEDRO, Françoise, Trajectoires des Maîtres au cabotage des ports de la rade de Lorient au XVIIIe siècle : entre tradition et mobilité, dir. G. LE BOUËDEC.

\section{DEA}

2000

SEIGNARD, Anne-Laure, Le soldat sur le littoral breton (1715-1783), dir. G. LE BOUËDEC.

TouzE, Vincent,Les populations littorales face aux pouvoirs : le refus des pouvoirs dans le golfe $\mathrm{du}$ Morbihan au XVIIIe siècle, dir. G. LE BOUËDEC.

VINCENT,Johan,Les paradis de papier : les sociétés littorales face à l'intrusion balnéaire (XIXe siècle-1945) : Les sites selon les guides et les récits de voyage, dir. G. LE BOUËDEC.

\section{1}

AMGHAR,Julien, La construction portuaire et les activités maritimes dans le golfe du Morbihan du XVIIIe siècle au début du XXe siècle, dir. G. LE BOUËDEC.

AUDRAN,Karine, Les ports Bretons sous la Révolution, le Consulat et l'Empire, Saint-Malo, Morlaix, Brest, Quimper, Lorient, dir. G. LE BOUËDEC.

LE BIHAN,Jeannine, L'émergence de l'activité ostréicole dans le golfe du Morbihan, dir. G. LE BOUËDEC.

LE GOUGUEC,Anne-Françoise, Les mutations du cabotage au XIXe siècle d'après les rôles de bord des maîtres au cabotage des quartiers de vannes et d'Auray, dir. G. LE BOUËDEC.

\section{2}

BoIsson,Pascal, Les entreprises fondées par Emile Marcesche : Emile Marcesche (1868-1939), entrepreneur lorientais de grande envergure, dir. G. LE BOUËDEC.

COINDET, Sylvain, Gens de mer et naufrage littoral en Bretagne au XVIIIe siècle, dir. G. LE BOUËDEC.

LAUNAY,Anne-Gaëlle, Aspects de la Mayenne pendant la guerre franco-prussienne de 1870-1871, dir. G. LE BOUËDEC.

PICAUD, Marie-Aude,Les imprimeurs-libraires des villes-ports de Bretagne au XVIIIe siècle : fonds de librairie et profils des villes-ports, dir. G. LE BOUËDEC.

SPELLA, Nadège,Organisation et mutations du littoral mecklembourgeois, 1790-1820, dir. G. LE BOUËDEC. 


\section{3}

BRIENT, Xavier,L'Armement au cabotage en Bretagne-Sud (1815-1848), dir. G. LE BOUËDEC.

MÉNARD, Marie,Autopsie de la première Compagnie des Indes Orientales (1664-1719), dir. G. LE BOUËDEC. PERRIN, Michel,L'Armement maritime à Lorient et dans sa région (1740-1940), dir. G. LE BOÜ̈DEC. ROUELLE, Martine, Les notaires du littoral du Morbihan et la réforme du notariat : (de la fin de l'Ancien Régime à l'Empire), dir. G. LE BOUËDEC.

\section{Master 2}

\section{4}

LE BOUËDEC, Anthony Les élites municipales d'Hennebont et de Lorient de la fin de l'Ancien Régime à la Révolution (1770-1799), dir. G. LE BOUËDEC.

MOURIES, Damien, Les milieux négociants français et le commerce intraeuropéen au XVIIe siècle, dir. G. LE BOUËDEC.

\section{5}

FAUCHILLE, Gwénaël,Les processus de patrimonialisation dans les petits ports de pêche artisanale sur la côte nord du Portugal, dir. G. LE BOUËDEC.

GUIHUR, Evelyne,L'Asie et les vocations des missionnaires bretons de la Société des Missions étrangères de Paris (1660-1791) : des vocations sacerdotales ordinaires ?, dir. G. LE BOUËDEC.

LE DOUDIC, Kévin,Les Français dans l'Océan Indien au temps de la Compagnie des Indes : culture(s) et cadre de vie social et matériel, dir. G. LE BOUËDEC.

LE RUYET, Louis, Migrations et déplacements des immatriculés, marins et ouvriers spécialisés sans la construction denavires, de 1666 à la Révolution, dans les quartiers de Port-Louis et Lorient ; habitués et sédentarisésdans les paroisses riveraines, dir. G. LE BOUËDEC.

MALONDA, Frédéric,Les pêcheurs et le service à l'État dans la marine de guerre (1880-1900), dir. G. LE B OUËDEC.

PICAUD, Marie-Aude,Les imprimeurs-libraires des villes-ports de Bretagne au XVIIIe siècle : fonds de librairie et profils des villes-ports, dir. G. LE BOUËDEC.

\section{6}

CATROU, André, Les élites consulaires de Caen, Morlaix et Alençon au XVIIIe siècle (1710-1792), dir. G. LE B OUËDEC.

MARGOLINE-PLOT, Eugénie, La diffusion de la culture asiatique en Bretagne au XVIIIe siècle, Pacotilles et commerces illicites, dir. G. LE BOUEDEC.

NOFFICIAL, sébastien,Les ports-arsenaux bretons face aux guerres maritimes de la Révolution, Brest et Lorient au printemps 1793, dir. G. LE BOUEDEC. 


\section{7}

FROHNMAIER, Gudrun, Les Français à Hambourg : négociants, émigrés, citoyens. Hambourg et la France: administrés, administrateurs (1789-1814), dir. G. LE BOUËDEC.

GREMARE, Benoît,La militarisation de la Bretagne (1661-1789), dir. G. LE BOUEDEC.

LANGLOIS, Ludivine,Les Circuits du cabotage en Bretagne au XVIIIe siècle. Approche méthodologique (étude des liens portuaires entre Nantes, Lorient et Quimper de 1753 à 1755), dir. G. LE BOUEDEC.

\section{Thèses}

2002

DuBoIs, Xavier, Les mutations des systèmes sardiniers en Bretagne-Sud au XIXe siècle, dir. G. LE BOUEDEC. FERNANDEZ DOMINGO, Enrique, Les relations économiques entre la France et le Chili, 1880-1929. Une étude sur le négoce français au « cono sur » américain, dir. O. PETRE-GRENOUILLEAU.

2003

POURCHASSE, Pierrick, Le commerce du Nord. Les échanges commerciaux entre la France et l'Europe septentrionale au XVIIIe siècle, dir. G. LE BOUEDEC.

2004

MARTIN, Pierre, Les fermiers du rivage. Droits maritimes, seigneurs, fermiers et fraudeurs en Bretagne sous l'Ancien Régime, dir. G. LE BOUEDEC.

\section{5}

VINCENT, Johan,Les sociétés littorales face à l'intrusion balnéaire, de Morgat à la Faute-sur-Mer (début XIXe siècle-1945), dir. G. LE BOUEDEC.

2006

AMGHAR, Julien, Les petits ports et les usages du littoral en Bretagne au xixe siècle, dir. G. LE BOUËDEC. PENCALET-KERIVEL, Françoise, « Les Mauritaniens » de 1945 à 1990, dir. G. LE BOUËDEC.

\section{7}

AUDRAN, Karine, Les négoces portuaires sous la Révolution et l'Empire. Bilan et stratégies. Saint-Malo, Brest, Lorient et Nantes, 1789-1815, dir. G. LE BOUËDEC

LE BIHAN, Jeannine, Ostréiculture et société ostréicole en Bretagne-Sud (1850-1986), dir. G. LE BOUËDEC. VARY, Morgane, Intégration sociale des populations marginales sur le littoral breton au xviiie siècle, dir. G. LE BOUËDEC. 
INDEX

Index géographique : Lorient 